\title{
A new gamma-ray source unveiled by AGILE in the region of Orion
}

\author{
N. Marchili ${ }^{1}$, G. Piano ${ }^{1}$, M. Cardillo ${ }^{1}$, A. Giuliani $^{2}$, S. Molinari ${ }^{1}$, and M. Tavani ${ }^{1}$ \\ ${ }^{1}$ INAF - IAPS, Via Fosso del Cavaliere 100, 00133 Roma, Italy \\ e-mail: nicola.marchili@gmail.com \\ 2 INAF - IASF Milano, Via E. Bassini 15, 20133 Milano, Italy
}

Received 21 November 2017 / Accepted 14 March 2018

\begin{abstract}
Context. Diffuse galactic $\gamma$-ray emission is produced by the interaction of cosmic rays (CRs) with the interstellar environment. The study of $\gamma$-ray emission is therefore a powerful tool that can be used to investigate the origin of CRs and the processes through which they are accelerated.

Aims. Our aim is to gain deeper insights into the nature of $\gamma$-ray emission in the region of Orion, which is one of the best studied sites of ongoing star formation, by analysing data from the AGILE satellite. Because of the large amount of interstellar medium (ISM) present in it, the diffuse $\gamma$-ray emission expected from the Orion region is relatively high. Its separation from the galactic plane also ensures a very small contribution from foreground or background emission, which makes it an ideal site for studying the processes of particle acceleration in star-forming environments.

Methods. The AGILE data are modelled through a template that quantifies the $\gamma$-ray diffuse emission expected from atomic and molecular hydrogen. Other sources of emission, such as inverse Compton (IC) scattering in interstellar radiation fields (ISRF) and extragalactic background, can be modelled as an isotropic contribution.

Results. Gamma-ray emission exceeding the amount expected by the diffuse emission model is detected with a high level of significance. The main excess is in the high-longitude part of Orion A, which confirms previous results from the Fermi Large Area Telescope. A thorough analysis of this feature suggests a connection between the observed $\gamma$-ray emission and the B0.5 Ia star $\kappa$ Orionis.

Conclusions. We present the results of the investigation of $\gamma$-ray diffuse galactic emission from the region of Orion. The comparison between modelled and observed emission points towards the existence of higher-than-expected $\gamma$-ray flux from a $1^{\circ}$ radius region centred in $\kappa$ Orionis, compatible with the site where stellar wind collides with the ISM. Scattering on dark gas and cosmic-ray acceleration at the shock between the two environments are both discussed as possible explanations, with the latter hypothesis being supported by the hardness of the energy spectrum of the emission. If confirmed, this would be the first direct detection of $\gamma$-ray emission from the interaction between ISM and a single star's stellar wind.
\end{abstract}

Key words. acceleration of particles - stars: winds, outflows - cosmic rays - gamma rays: ISM - ISM: individual objects: Orion A

\section{Introduction}

More than a century after the discovery of cosmic rays (CRs), the huge effort put into investigating their origin and the mechanisms of their acceleration has provided us with a basic picture that still leaves open some fundamental questions.

Convincing arguments support the thesis that shocks of young supernova remnants are the main site of CR acceleration in the Galaxy (Blandford \& Eichler 1987). Their isotopic and elemental abundances suggest that $\mathrm{CR}$ nuclei are a mixture of $\sim 20 \%$ ejecta from high-mass stars and $\sim 80 \%$ gas from the interstellar medium (ISM; Binns et al. 2005; Rauch et al. 2009). The contribution of high-mass stars to the composition of CRs is therefore substantial; it is less clear, instead, how important their contribution is to the acceleration of particles. High-mass stars tend to cluster in large associations hosted by molecular clouds. Here, the colliding winds may create shock waves. Diffusive shock acceleration has been invoked as the most likely process for the acceleration of CRs (Berezhko \& Ksenofontov 1999); in the case of high-mass star clusters, CR acceleration could take place as a consequence of the interaction among stellar winds (Bykov \& Toptygin 2001). There is strong evidence supporting the hypothesis that the $\gamma$-ray emission detected by the Fermi Large Area Telescope (LAT) in the Cygnus Superbubble is due to freshly accelerated CRs within the cavities carved by stellar winds from high-mass stars (Ackermann et al. 2011).

Munar-Adrover et al. (2011) cross-checked the Fermi First Source Catalog (Abdo et al. 2010) with catalogues of massive young stellar objects (MYSOs), Wolf-Rayet and Of-type stars, and $\mathrm{OB}$ associations and found significant spatial coincidence between $\gamma$-ray sources and MYSOs. This indicates that CR acceleration could also take place around single stellar objects when they form jets that could interact with the surrounding material. Casse \& Paul (1980) hypothesised that acceleration of CRs could also be triggered by OB stars in shocked regions at the boundary between ISM and the stellar wind. Voelk \& Forman (1982) investigated this case further and reached the conclusion that stellar winds might be sources of low-energy CRs caused by the acceleration of nuclear particles, while higher energies could only be achieved through the contribution of electrons provided that they can efficiently be injected and accelerated at the shock. The $\gamma$-ray luminosity from these regions is expected to be low.

In this paper we present the analysis of $\gamma$-ray data concerning the diffuse emission towards Orion, which is the nearest 
and most frequently observed site of ongoing star formation (see Bally 2008 and references therein), and neighbouring regions. The data were collected by the Gamma-Ray Imaging Detector (GRID, Barbiellini et al. 2002; Prest et al. 2003), which is the silicon-tracker imager on board the $\gamma$-ray satellite AGILE (Tavani et al. 2009). Orion is embedded in the OrionEridanus superbubble; it comprises the stellar association Orion OB1 and the giant molecular clouds Orion A and Orion B. Given its importance for the study of massive star formation mechanisms and its relatively high distance from the galactic plane, which reduces the contamination from foreground and background emission, the region of Orion has been the target of intensive high-resolution observations over a wide range of frequencies; in particular, because of its high concentration of molecular hydrogen, systematic observations have been performed in the $\mathrm{mm}$ to infrared bands, where $\mathrm{CO}$ abundances could be traced and turned into $\mathrm{H}_{2}$ abundances using the $\mathrm{X}_{\mathrm{CO}}$ conversion factor.

In the $\gamma$-ray band, the first detection of Orion belongs to COS-B (Caraveo et al. 1980), followed by EGRET in the 1990s (Digel et al. 1995). The sensitivity and spatial resolution reached by Fermi allowed a thorough study of the distribution of $\gamma$-ray emission from the whole region (Ackermann et al. 2012). The detection of an excess of $\gamma$-ray emission from the highlongitude part of Orion A $\left(1>212^{\circ}\right)$ was interpreted in terms of a non-linear relation between $\mathrm{H}_{2}$ and $\mathrm{CO}$ densities in diffuse molecular clouds. Our results confirm the findings of Fermi regarding the excess of $\gamma$-ray emission; however, we propose a different interpretation of its origin.

This paper is structured as follows: in Sect. 2 we describe the data and their calibration procedures; in Sect. 3 we explain the modelling of the galactic diffuse $\gamma$-ray emission; the difference between the best fit model and the AGILE data is presented in Sect. 4; in Sect. 5 we discuss the possible origin of the detected $\gamma$-ray excess; the summary is presented in Sect. 6 .

\section{Observations and data calibration}

We analysed AGILE-GRID data in the area highlighted in Fig. 1 as the region of interest $(\mathrm{ROI})$, a $15^{\circ} \times 11^{\circ}$ rectangle centred in $(l, b)=[210.5,-15.5]$. The data integrate observations performed by AGILE in the time span from November 2009, when the satellite started operating in spinning mode, to March 2017. The $\gamma$-ray energy range to which AGILE-GRID is sensitive goes from $30 \mathrm{MeV}$ to $30 \mathrm{GeV}$. The point spread function (PSF) at $100 \mathrm{MeV}$ is $4.2^{\circ}$, while at $400 \mathrm{MeV}$ it is $1.2^{\circ}$ (68\% containment radius; see Sabatini et al. 2015).

The AGILE-GRID data were analysed using the Build_22 scientific software, while calibrated filter and response matrices were respectively analysed with FM3.119 and I0025. The standard configuration was applied to the AGILE data analysis of incoming photons, which applies South Atlantic Anomaly event cuts and $80^{\circ}$ Earth albedo filtering; all incoming $\gamma$-ray photons with an off-axis higher than $60^{\circ}$ have been discarded.

The AGILE multi-source likelihood analysis (MSLA) software (Bulgarelli et al. 2012) provided the tools for the calculation of statistical significance and flux determination for both point-like and extended sources. This software is based on the likelihood analysis developed by Mattox et al. (1996) for EGRET data, which assesses the reliability of a source detection by looking at the ratio of the maximum-likelihood values of two alternative hypotheses, namely a no-source model,

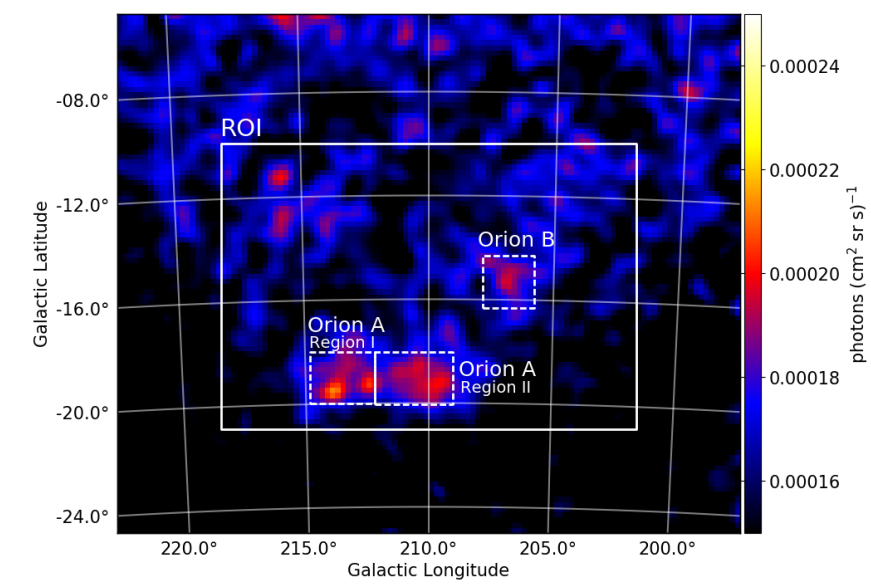

Fig. 1. AGILE map of Orion. The large continuous rectangle highlights the region of interest over which the best fit model was computed. From left to right, the dashed rectangles show the Region I and Region II subregions of Orion A, and Orion B.

to another that postulates the existence of sources in the field.

Within the ROI, following the example of Ackermann et al. (2012), we defined the three subregions Orion A, Region I; Orion A, Region II; and Orion B, which refer, respectively, to the giant molecular cloud Orion $\mathrm{A}$ and $\mathrm{B}$, the former being divided into two separate zones. These are shown as dashed rectangles in Fig. 1. In order to reduce the noise, a three-point smoothing has been applied to the data.

\section{Data modelling}

\subsection{Galactic diffuse emission}

Following the approach described in Giuliani et al. (2004), the galactic $\gamma$-ray diffuse emission as a function of galactic coordinates $(l, b)$, distance to the emitting medium $(r)$, and photon energy $(E), g(l, b, r, E)$, was modelled as

$$
\begin{aligned}
g(l, b, r, E)= & {\left[q_{\mathrm{pp}}(l, b, r, E)+q_{\mathrm{br}}(l, b, r, E)\right]\left[n_{\mathrm{HI}}(l, b, r)\right.} \\
& \left.+2 n_{\mathrm{H}_{2}}(l, b, r)\right]+q_{\mathrm{IC}}(l, b, r, E) n_{\mathrm{ph}}(l, b, r),
\end{aligned}
$$

where $q_{\mathrm{pp}}$ and $q_{\mathrm{br}}$ are the $\gamma$-ray emissivities per hydrogen atom due to proton-proton scattering and Bremsstrahlung, respectively; $q_{\mathrm{IC}}$ is the $\gamma$-ray emissivity per target photon due to inverse-Compton (IC) scattering; $n_{\mathrm{HI}}, n_{\mathrm{H}_{2}}$, and $n_{\mathrm{ph}}$ are the densities of atomic hydrogen, molecular hydrogen, and interstellar radiation field (ISRF) photons.

The HI distribution in the galaxy was retrieved from the Leiden/Argentine/Bonn (LAB) 21-cm Survey (see Kalberla et al. 2005; Hartmann \& Burton 1997). The $\mathrm{H}_{2}$ column densities, which cannot be determined through direct observation, were assessed using the CO survey described by Dame et al. (2001); the $\mathrm{CO}$ has been converted into $\mathrm{H}_{2}$ abundance using a CO-to- $\mathrm{H}_{2}$ conversion factor, $\mathrm{X}_{\mathrm{CO}}$. Due to the small variations of the ISRF across the region of Orion, the IC contribution to the total $\gamma$-ray emission, which in the ROI is marginal, was approximated as an isotropic component.

The comparison between the modelled galactic $\gamma$-ray diffuse emission and the AGILE maps required the $g$ dependence on $r$ and $E$ to be removed. For this work, the whole sky was divided into squares of $0.1 \times 0.1 \mathrm{deg}^{2}$; the $g(l, b)$ values we analysed were 

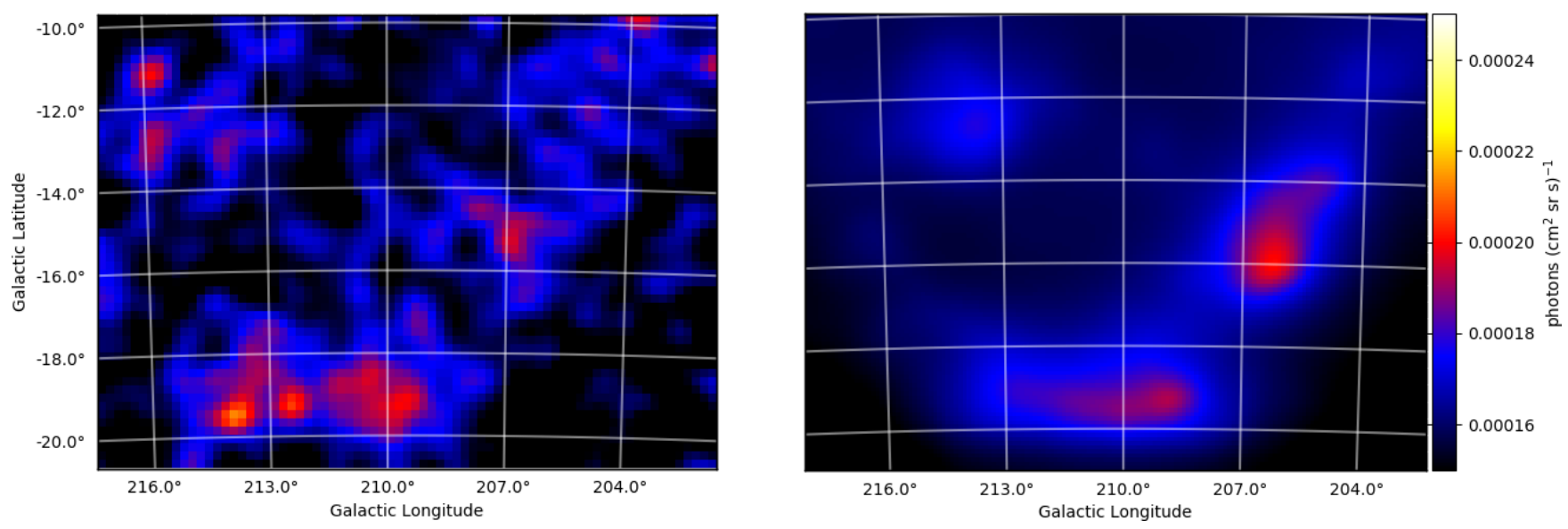

Fig. 2. Comparison between the AGILE (left panel) and the modelled (right panel) maps of the Orion region.

obtained by integrating $g(l, b, r, E)$ over distance and over the energy range 100-50 $000 \mathrm{MeV}$.

\subsection{Complete model}

Galactic diffuse emission is not the only source of $\gamma$-ray photons in the AGILE bandwidth. The $\gamma$-ray flux detected by AGILE also comprises point-like sources and isotropic extragalactic background. Given that the Second AGILE-GRID Source catalogue does not contain any objects in the ROI, the contribution from the former ones can be ignored. The extragalactic contribution, instead, can be modelled as an isotropic component.

A piece of software was developed to provide us with the best fit to the complete emission model presented above. It allows us to separately treat the contributions that $\mathrm{HI}$ and $\mathrm{H}_{2}$ provide to the observed $\gamma$-ray sky $\left(S_{\mathrm{HI}}(l, b)\right.$ and $S_{\mathrm{H}_{2}}(l, b)$, respectively).

The total AGILE flux $S_{\text {tot }}$ was fitted either on the whole ROI or on specific subregions as a linear combination of $S_{\mathrm{HI}}$ and $S_{\mathrm{H}_{2}}$,

$S_{\text {tot }}(l, b)=\alpha S_{\mathrm{HI}}(l, b)+\beta S_{\mathrm{H}_{2}}(l, b)+\epsilon$,

where the best fit proportionality factors $\alpha$ and $\beta$ were calculated through minimisation of least squares, along with the isotropic component $\epsilon$ (which also includes, as explained above, the IC contribution). The results of this modelling procedure are displayed in Fig. 2, where the AGILE map (left panel) is shown together with the best fit model (right panel).

\section{Results}

The analysis of the AGILE data has important implications for both the model and the investigation of diffuse $\gamma$-ray emission. For the former, a significant part of the emission from the ROI is expected to come from molecular hydrogen. The comparison of the modelled and the measured $\gamma$-ray fluxes provides us with an interesting method for estimating the $\mathrm{X}_{\mathrm{CO}}$ factor. Also, the detection of localised flux excess can be used to reveal regions where the model fails, hinting at the presence of dark gas, at a non-linear relation between $\mathrm{H}_{2}$ and $\mathrm{CO}$ densities, or even at overabundances of CRs.

\subsection{Estimation of the $X_{C O}$ factor}

The calculation of the best fit proportionality factors $\alpha$ and $\beta$ allows us to estimate the conversion factor $\mathrm{X}_{\mathrm{CO}}$ by comparing the contributions of $S_{\mathrm{HI}}$ and $S_{\mathrm{H}_{2}}$ to the total flux. Assuming that $n_{\mathrm{HI}}$ and $n_{\mathrm{H}_{2}}$ were both correctly calculated, we would expect $\alpha$ and $\beta$ to have the same value. A difference between the two values, instead, would naturally be attributed to the highest source of uncertainty in the column density estimators, namely the conversion factor $\mathrm{X}_{\mathrm{CO}}$. The effective conversion factor, $\left(\mathrm{X}_{\mathrm{CO}}\right)_{\mathrm{eff}}$, could then be calculated as

$\left(\mathrm{X}_{\mathrm{CO}}\right)_{\mathrm{eff}}=\frac{\beta}{\alpha} \mathrm{X}_{\mathrm{CO}}$

This computation method leads to $\left(\mathrm{X}_{\mathrm{CO}}\right)_{\mathrm{eff}}=(1.32 \pm 0.05) \times$ $10^{20} \mathrm{~cm}^{-2} \mathrm{~K}^{-1} \mathrm{~km}^{-1} \mathrm{~s}$, where the error refers to the statistical uncertainty in the computation of $\alpha$ and $\beta$. It should be noted, though, that larger uncertainties may be introduced in the $\left(\mathrm{X}_{\mathrm{CO}}\right)_{\text {eff }}$ calculation by approximations in the templates of the HI and $\mathrm{H}_{2}$ contributions to the $\gamma$-ray emission, $S_{\mathrm{HI}}$ and $S_{\mathrm{H}_{2}}$. These approximations could cause a systematic error of up to $30 \%$ in the estimation of $\left(\mathrm{X}_{\mathrm{CO}}\right)_{\mathrm{eff}}$.

The $\left(\mathrm{X}_{\mathrm{CO}}\right)_{\text {eff }}$ value we obtained is in excellent agreement with previous measurement provided by Fermi and EGRET, respectively $(1.36 \pm 0.02) \times 10^{20}$ (see Ackermann et al. 2012) and $(1.35 \pm 0.15) \times 10^{20} \mathrm{~cm}^{-2} \mathrm{~K}^{-1} \mathrm{~km}^{-1} \mathrm{~s}$ (Digel et al. 1999).

\subsection{Excess of $\gamma$-ray emission around $(l, b)=[214.4,-18.5]$}

The best fit model from Eq. (2) seems to fit the AGILE data very well. After removing the isotropic extragalactic component, most of the detected $\gamma$-ray diffuse emission in the map can be attributed to the molecular hydrogen clouds in the region of Orion, with a fainter contribution coming from atomic hydrogen. However, a careful examination of the residuals from the fitted model reveals some excess in emission not accounted for by our template (see Fig. 3).

The most prominent feature is an overabundance of $\gamma$-ray photons in Region I of Orion A. Most of the emission is concentrated in a moderately extended spot whose approximate position is $(l, b)=[213.9,-19.5]$. This result confirms a similar analysis carried out by Ackermann et al. (2012) on Fermi LAT data, showing that, also at the lower tail of energy where AGILE is most sensitive, the currently available atomic and molecular hydrogen distributions cannot fully explain the whole diffuse emission in the Orion region.

We performed likelihood analyses of the detected excess of $\gamma$-ray flux under the assumptions of both extended and point-like emission. In the hypothesis of extended emission, the detection 
is significant at a $5.2 \sigma$ level, with an estimated flux of $11 \pm 2 \times$ $10^{-8}$ photons $\mathrm{cm}^{-2} \mathrm{~s}^{-1}$, while in the case of a point-like source, the analysis returns a detection significance at a $4.4 \sigma$ level, and an estimated flux of $6.8 \pm 1.6 \times 10^{-8}$ photons $\mathrm{cm}^{-2} \mathrm{~s}^{-1}$. These results, which moderately favour the hypothesis of extended emission, do not allow us to identify its origin with certainty; this is not surprising given that the PSF of AGILE is almost as large as the analysed feature. In this respect, it should be noted that the detection of a point-like feature in our maps does not imply that the source of the emission is point-like; however, the assumption of an extended source of emission is strongly supported by several arguments. The Fermi analysis of the same spot also suggested an extended emission; there are no counterparts at different wavelengths to support the idea of a point-like source. Finally, it appears that the excess emission discussed above is not isolated; it seems to be part of an extended, continuous region approximately shaped like an arc.

To assess the importance of the arc-shaped feature, which is hardly compatible with a point-like source, with respect to analogous extended structures that may be present in the map of residuals, we developed a script that integrates the residual flux over circles of variable size, centred on any point of the ROI. For the whole set of integrated fluxes it calculates the average and standard deviation. The only feature with integrated flux reaching approximately a $5 \sigma$ detection is a circle with $1^{\circ}$ radius centred in $(l, b)=[214.4,-18.5]$ (see the white circle in Fig. 3). The nature of this emission (which, according to the arguments above, from now on is assumed to be extended) is thoroughly discussed in Sect. 5. A further but less significant source of $\gamma$-ray excess can be found at the centre of this circle.

\subsection{Unidentified source in $(l, b)=[215.8,-11.2]$}

Another interesting feature of the map of residuals is the excess of emission around $(l, b)=[215.8,-11.2]$ (see green dot in Fig. 3), which is significant to a $3.9 \sigma$ level, with an estimated flux of $4.4 \pm 1.2 \times 10^{-8}$ photons $\mathrm{cm}^{-2} \mathrm{~s}^{-1}$. It can most likely be associated with the EGRET source 3EG J0616-0720, classified as an unidentified object, whose coordinates in the Third EGRET Catalog (Hartman et al. 1999) are $(l, b)=[215.58,-11.06]$, with an uncertainty on the position of $0.6-0.9^{\circ}$. It should be noted that 3EG J0616-0720 is among the 107 sources that have not been inserted into the EGret Revised (EGR) source list (Casandjian \& Grenier 2008) because of additional structure in the interstellar background. This leaves open the question whether the emission should be associated with a point-like source or with extended structures in the ISM.

\section{Discussion}

\subsection{Integrating dark gas tracers into the model}

Following Grenier et al. (2005), local excesses of $\gamma$-ray diffuse emission can be interpreted as the result of the interaction between CRs and clouds of cold dust and dark gas that are not traced by atomic hydrogen and $\mathrm{CO}$ surveys. We assessed the possibility of improving our fit of the $\gamma$-ray diffuse emission detected by AGILE by integrating dark gas tracers into a new model, which can be expressed as

$S_{\text {tot }}(l, b)=\alpha S_{\mathrm{HI}}(l, b)+\beta S_{\mathrm{H}_{2}}(l, b)+\gamma S_{\mathrm{DG}}(l, b)+\epsilon$,

where $S_{\mathrm{DG}}(l, b)$ is an estimate of $\gamma$-ray radiation from the interaction of dark gas with CRs.

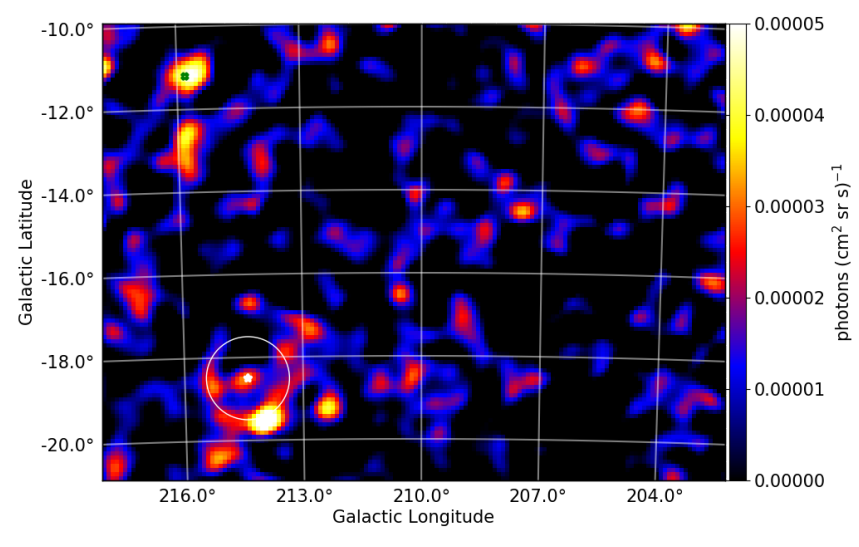

Fig. 3. Residuals of the best fit diffuse emission model to the AGILE map. The white circle shows the ring of $\gamma$-ray photon excess centred on $\kappa$ Orionis (white star). The green dot on the top-left part of the map highlights the excess of emission corresponding to the unidentified EGRET source 3EG J0616-0720.

For the calculation of $S_{\mathrm{DG}}(l, b)$ three different templates of dark gas were created, which use as starting points the Planck $353 \mathrm{GHz}$ map (hereafter tracer A), the reddening map derived by IRAS and COBE data by Schlegel et al. (1998; hereafter tracer B), and the reddening map derived from Pan-STARRS1 stellar photometry by Schlafly et al. (2014; hereafter tracer C). For all three maps, the same procedure was followed for the estimation of dark gas clouds in the region of Orion 2:

- Column densities were derived from the original maps. The procedure for the conversion of the Planck map follows the approach of Kauffmann et al. (2008). For the extinction-to-column density conversion, we applied instead the conversion factor from Güver \& Özel (2009);

- Atomic and CO-traced molecular hydrogen contributions were subtracted from the column densities. Given the degree of uncertainty in the conversion of the original data into column densities, we note that proportionality factors were applied to the $\mathrm{HI}$ and $\mathrm{H}_{2}$ contributions in order to minimise the residuals of the subtraction. This means that the degree of correlation between the spatial distributions of $\mathrm{HI} / \mathrm{H}_{2}$ and dark gas is also minimised;

- The residuals of the subtraction were retained as templates of dark gas.

The choice of minimising the correlation between the spatial distributions of $\mathrm{HI} / \mathrm{H}_{2}$ and dark gas may have some minor effect on the estimation of the best fit parameters of the model; for example, part of the contribution due to dark gas could be accounted for as a $\mathrm{H}_{2}$ contribution. This, however, should not affect the calculation of residuals of the new modelling.

The three tracers of dark gas are shown in Fig. 4. While tracer A shows very localised regions of dark gas (left panel), with almost no correlation with the $\gamma$-ray excess in Fig. 3, the tracers inferred from reddening maps suggest an extended distribution of dark gas in Region I of Orion A. In the case of tracer C (right panel), the distribution of dark gas is mainly concentrated around a ring approximately centred at $(l, b)=[214.4,-18.5]$, which makes it the most interesting candidate for explaining the $\gamma$-ray excess detected by AGILE.

We therefore used tracer $\mathrm{C}$ as a template for $S_{\mathrm{DG}}(l, b)$, and recalculated the best fit model from Eq. (4). The residuals of the improved modelling are shown in Fig. 5. It may be surprising to see that the similarities between the dark gas tracer and the AGILE $\gamma$-ray excess do not translate into a substantial reduction 

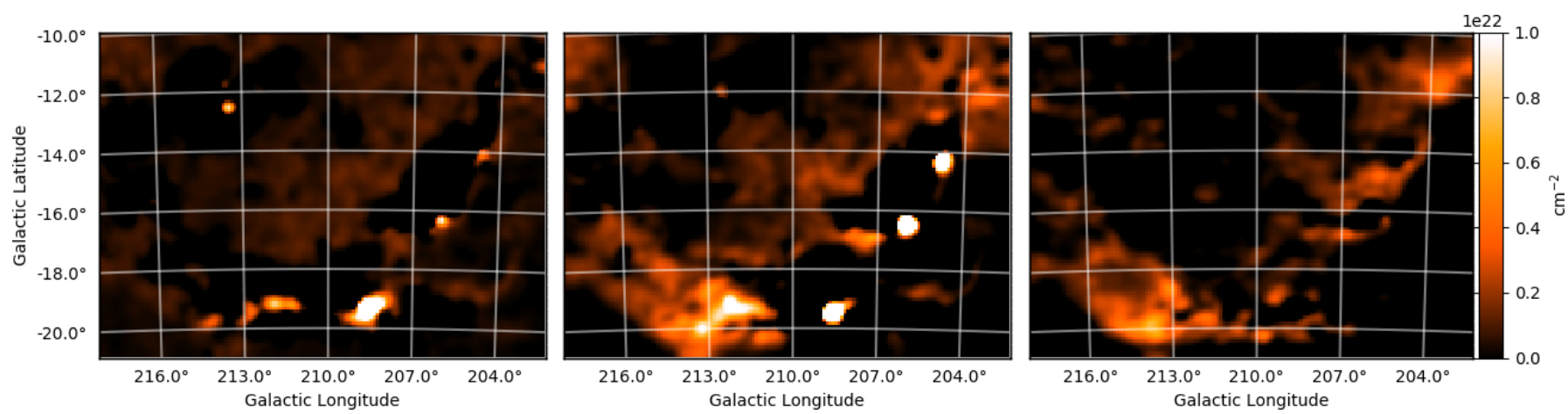

Fig. 4. Column densities of dark gas estimated from the Planck $353 \mathrm{GHz}$ map (left panel), the reddening map by Schlegel et al. (1998; middle panel), and the one by Schlafly et al. (2014; right panel).

of the excess flux. The only noticeable difference with respect to the model expressed in Eq. (2) is a $\sim 25 \%$ decrease in the residual of $\gamma$-ray flux in $(l, b)=[213.9,-19.5]$. The relatively small impact of the improved model on the fit can be explained with some significant difference in the distribution of the dark gas with respect to the diffuse $\gamma$-ray emission. Both dark gas and $\gamma$-ray estimates point towards the existence of a ring around $(l, b)=[214.4,-18.5]$; however, the diameter of the ring and the distribution of the material/emission along the ring are not the same for the two cases. Apparently, either our dark gas tracers are not able to detect all the medium in Region I of Orion A or the $\gamma$-ray excess is not due to dark gas, but rather to a localised overabundance of CRs.

\section{2. $\kappa$ Orionis ring}

As mentioned above, the ring-shaped excess of $\gamma$-ray photons in Region I of Orion A has approximately the same centre $(l, b)=[214.4,-18.5]$ as the ring in our dark gas tracer C. Considering the approximation due to the smoothing of the AGILE map and the uncertainty in the reconstruction of the origin of gamma rays, the centre of the ring is compatible with the position of $\kappa$ Orionis $(l, b)=[214.5,-18.5]$. This is a blue supergiant star of spectral type B0.5 Ia, which parallax measurements place at a distance of about $200 \mathrm{pc}$ (see van Leeuwen 2007). There is evidence supporting the hypothesis that this association is not a pure coincidence. A recent work by Pillitteri et al. (2016) found strong hints of the existence of a star-forming ring of 5$8 \mathrm{pc}$ radius around $\kappa$ Orionis which the authors identified by looking at the X-ray luminosity function of the young stellar objects (YSO) detected in two fields between L1641 S and $\kappa$ Orionis. They hypothesised that the ring is part of a shell in which the gas and the dust from the Orion-Eridanus superbubble have been swept up by the strong winds rising from the star, triggering star formation at the edge of the shell. The same shell was reported in near-IR extinction maps from the Two Micron All Sky Survey (2MASS, see Kleinmann et al. 1994; Lombardi et al. 2011); it is also visible in the CO maps from Dame et al. (2001), and all ESA Planck maps between 217 and $857 \mathrm{GHz}$.

The $\gamma$-ray ring, with a radius of about $4 \mathrm{pc}$, could therefore be seen as the inner border of the star-forming shell revealed by Pillitteri et al. (2016). This interpretation is supported by the comparison between the AGILE map and the $\mathrm{CO}$ emission from Dame et al. (2001) shown in Fig. 6.

The existence of two concentric shells can be explained within coherent physical frameworks. Two competing scenarios seem plausible: a high concentration of dark gas and locally accelerated CRs. In terms of dark gas, the winds rising from $\kappa$ Orionis could push the surrounding gas and dust towards the outside; as the temperature decreases and the density of the medium increases with distance from the star, molecular hydrogen could be formed. On the inner edge, the UV radiation that the star emits would prevent the formation of $\mathrm{CO}$, which therefore would no longer trace the molecular hydrogen shell. However, its interaction with CRs would reveal it as an excess of $\gamma$-ray photons. Farther from the star, the temperature and density of the medium, together with the lower flux of UV radiation, could provide the conditions for the formation of molecular clouds where star formation could be triggered.

Locally accelerated CRs could provide us with a different interpretation. It involves the particle acceleration process hypothesised by Casse \& Paul (1980) for the shocked regions where stellar wind interacts with the ISM. The acceleration of CRs in this site would naturally translate into an increase in $\gamma$-ray emission. Interestingly, the authors suggest Orion as an ideal candidate for testing their hypothesis, because of the high flux expected from the region. We calculated the presumable $\gamma$-ray flux from around $\kappa$ Orionis using the same model the authors proposed for $\rho$ Ophiuchi: $F_{\gamma}(\geq 100 \mathrm{MeV})=$ $10^{-6} \mathrm{M} \epsilon / d^{2}$ photons $\mathrm{cm}^{-2} \mathrm{~s}^{-1}$, where $M$ is the total mass of the dense cloud in $M_{\odot}, \epsilon$ is the local $\gamma$-ray emissivity in $10^{-25}$ photons $\left(\geq 100 \mathrm{MeV}^{-1} \mathrm{~s}^{-1} \text { (H-atom }\right)^{-1}$, and $d$ is the distance to the source in pc. Through the Planck function we converted the Planck $857 \mathrm{GHz}$ flux from within the $4 \mathrm{pc}$ ring around $\kappa$ Orionis into an estimate of the mass $M$ of the cloud, obtaining a value of $1-1.5 \times 10^{3} M_{\odot}$. We estimated $\epsilon$ to be approximately 3 and, using for $\kappa$ Orionis a distance of $200 \mathrm{pc}$, we were able to calculate an $F_{\gamma}$ of the order of $10^{-7}$ photons $\mathrm{cm}^{-2} \mathrm{~s}^{-1}$, which is consistent with the flux estimate from our AGILE data. We note that, according to Voelk \& Forman (1982), stellar winds would primarily be sources of nuclear CRs; electron acceleration would be less favoured, which would explain why we did not find a radio counterpart for the $\gamma$-ray emission in the VLA maps of the region.

The analysis of the energy spectrum from the $\kappa$ Orionis ring reveals a spectral index of $1.7 \pm 0.2$, indicating that the emission is hard. This suggests, as in the case of the Cygnus superbubble observed by Fermi (Ackermann et al. 2011), that the origin of the radiation is more likely a population of freshly accelerated CRs. In our case, however, the acceleration would not be the result of the interaction between the stellar winds from a number of young stellar objects; it would arise as the consequence of the collision of the strong stellar wind from a single 


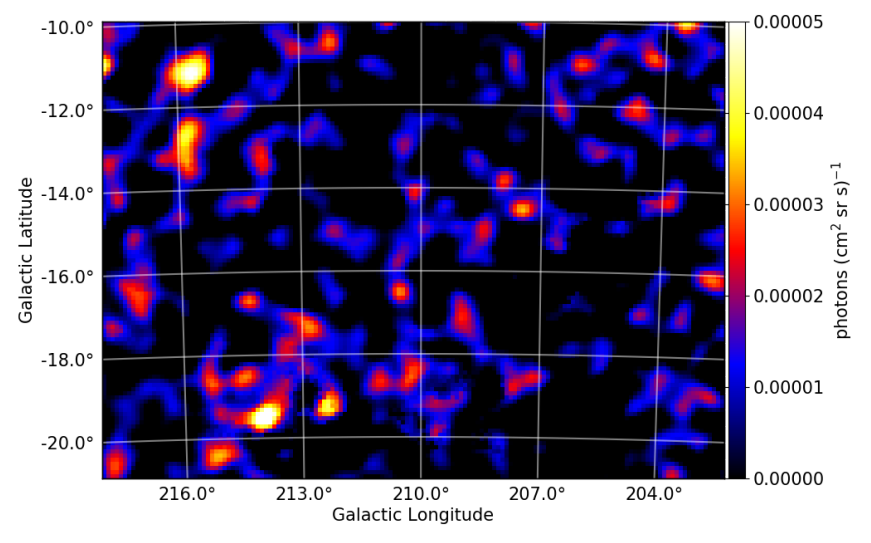

Fig. 5. Residuals of the best fit diffuse emission model to the AGILE map, after including the dark gas tracer $\mathrm{C}$ into the calculation.

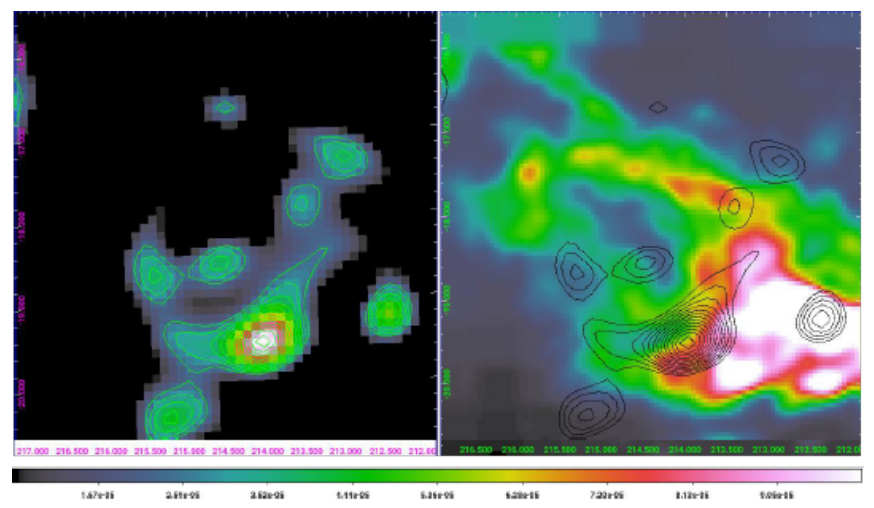

Fig. 6. Left panel: ring of $\gamma$-ray excess detected by AGILE. Right panel: CO map from Dame et al. (2001), which reveals the star-forming shell discussed by Pillitteri et al. (2016). The contour levels from the $\gamma$-ray data are shown in black.

star with the surrounding ISM. The accurate modelling of this process is still being developed and will be presented in a future publication.

\section{3. $\kappa$ Orionis point-like emission}

Another feature of $\kappa$ Orionis that deserves some attention is the mild excess of $\gamma$-ray emission within the ring surrounding this star. It belongs to a point-like source whose position coincides with that of the B0.5 Ia star. A point-like likelihood analysis applied to the source returned a $4 \sigma$ detection with a flux of $6.2 \pm 1.6 \times 10^{-8}$ photons $\mathrm{cm}^{-2} \mathrm{~s}^{-1}$. The origin of this $\gamma$-ray emission is not certain yet. The existence of a link with $\kappa$ Orionis, given the coincidence between the positions of the $\gamma$-ray source and the star, is an intriguing speculation. In a theoretical work about IC scattering on ISRF around OB stars, Orlando $\&$ Strong (2007) indicated in $\kappa$ Orionis the most likely candidate for which $\gamma$-ray emission due to IC scattering could be detected by Fermi-LAT with at least one year of integration time. It can be hypothesised, therefore, that the origin of the $\gamma$-ray excess detected by AGILE is cosmic-ray IC scattering on the photons of the star. According to the model by Orlando \& Strong (2007), however, the emitted flux should be of the order of $10^{-9}$ photons $\mathrm{cm}^{-2} \mathrm{~s}^{-1}$, more than one order of magnitude lower than the value we detected. It is true that the flux values provided in the paper, according to the authors, are rather conservative; however, to justify the observed discrepancy we would need to assume a substantial difference between the modelled and the actual electron spectrum in the vicinity of $\kappa$ Orionis.

\section{Summary}

We analysed the diffuse $\gamma$-ray emission collected by AGILE in the region of Orion. The data, covering the time span from November 2009 to March 2017, were modelled using a template that takes into account proton-proton scattering and Bremsstrahlung emission for atomic and molecular hydrogen, while the contribution from both IC scattering on the ISRF and extragalactic background were approximated as an isotropic contribution. The model, which is characterised by three free parameters, proved to be efficient in the description of the $\gamma$-ray flux emitted in the Orion region. By looking at the best fit values of the free parameters, we calculated a $\mathrm{X}_{\mathrm{CO}}$ conversion factor of $(1.32 \pm 0.05) \times 10^{20} \mathrm{~cm}^{-2} \mathrm{~K}^{-1} \mathrm{~km}^{-1} \mathrm{~s}$, consistent with previous estimations by Fermi and EGRET.

The fit of the model to AGILE data allowed us to identify local spots where the $\gamma$-ray emission is higher than expected from the identified ISM content. A putative detection of $\gamma$ ray excess from the B0.5 Ia supergiant star $\kappa$ Orionis raises the intriguing hypothesis that we are observing the emission due to IC scattering on the ISRF around the star. The most interesting feature emerging from our analysis, however, is an arc-shaped excess which appears to belong to a ring centred on $\kappa$ Orionis itself. This confirms a previous detection of $\gamma$-ray excess from the Orion A region reported by the Fermi collaboration (Ackermann et al. 2012). We hypothesise that the ring, which has a radius of $\sim 4 \mathrm{pc}$, corresponds to the inner side of a larger ring, identified by Pillitteri et al. (2016) as a star-forming region associated with a shell of dense gas surrounding $\kappa$ Orionis. The excess of $\gamma$-ray emission around the star could be due to dark gas at the edge of the shell swept up by the star or to CR acceleration in shocked regions where the stellar wind collides with the ISM. The hardness of the energy spectrum from the $\gamma$-ray ring points towards the latter hypothesis. A more thorough analysis of the available data, including observations at different wavelengths, is ongoing; it will help us to accurately model the acceleration process triggered by stellar wind. If our hypothesis is confirmed, the $\kappa$ Orionis ring could represent the first direct detection of diffuse $\gamma$-ray emission caused by the interaction between the winds of a single OB star and the ISM.

Acknowledgements. We wish to thank the anonymous referee for the useful comments which significantly improved the paper. We would like to thank Dr. Kazi Rygl for the stimulating discussion and the helpful suggestions. NM acknowledges the support given to the research activity for this work by the VIALACTEA Project, a Collaborative Project under Framework Programme 7 of the European Union funded under Contract \#607380. AGILE is an ASI space mission developed with programme support by INAF and INFN. This study was carried out with partial support through ASI grant no. I/028/12/4.

\section{References}

Abdo, A. A., Ackermann, M., Ajello, M., et al. 2010, ApJS, 188, 405 Ackermann, M., Ajello, M., Allafort, A., et al. 2011, Science, 334, 1103 Ackermann, M., Ajello, M., Allafort, A., et al. 2012, ApJ, 756, 4

Bally, J. 2008, in Handbook of Star Forming Regions, Volume I, ed. B. Reipurth (San Francisco, CA: ASP), 459

Barbiellini, G., Fedel, G., Liello, F., et al. 2002, Nucl. Instrum. Methods Phys. Res. A, 490, 146

Berezhko, E. G., \& Ksenofontov, L. T. 1999, Zh. Eksp. Teor. Fiz., 89, 391 Binns, W. R., Wiedenbeck, M. E., Arnould, M., et al. 2005, ApJ, 634, 351 Blandford, R., \& Eichler, D. 1987, Phys. Rep., 154, 1

Bulgarelli, A., Chen, A. W., Tavani, M., et al. 2012, A\&A, 540, A79 
N. Marchili et al.: A new gamma-ray source unveiled by AGILE in the region of Orion

Bykov, A. M., \& Toptygin, I. N. 2001, Astron. Lett., 27, 625

Caraveo, P. A., Bennett, K., Bignami, G. F., et al. 1980, A\&A, 91, L3

Casandjian, J.-M., \& Grenier, I. A. 2008, A\&A, 489, 849

Casse, M., \& Paul, J. A. 1980, ApJ, 237, 236

Dame, T. M., Hartmann, D., \& Thaddeus, P. 2001, ApJ, 547, 792

Digel, S. W., Hunter, S. D., \& Mukherjee, R. 1995, ApJ, 441, 270

Digel, S. W., Aprile, E., Hunter, S. D., Mukherjee, R., \& Xu, F. 1999, ApJ, 520, 196

Giuliani, A., Chen, A., Mereghetti, S., et al. 2004, Mem. Soc. Astron. It., 5, 135

Grenier, I. A., Casandjian, J.-M., \& Terrier, R. 2005, Science, 307, 1292

Güver, T., \& Özel, F. 2009, MNRAS, 400, 2050

Hartmann, D., \& Burton, W. B. 1997, Atlas of Galactic Neutral Hydrogen (Cambridge: Cambridge University Press), 243

Hartman, R. C., Bertsch, D. L., Bloom, S. D., et al. 1999, ApJS, 123, 79

Kalberla, P. M. W., Burton, W. B., Hartmann, D., et al. 2005, A\&A, 440, 775

Kauffmann, J., Bertoldi, F., Bourke, T. L., Evans, II, N. J., \& Lee, C. W. 2008 A\&A, 487, 993
Kleinmann, S. G., Lysaght, M. G., Pughe, W. L., et al. 1994, Exp. Astron., 3, 65

Lombardi, M., Alves, J., \& Lada, C. J. 2011, A\&A, 535, A16

Mattox, J. R., Bertsch, D. L., Chiang, J., et al. 1996, ApJ, 461, 396

Munar-Adrover, P., Paredes, J. M., \& Romero, G. E. 2011, A\&A, 530, A72

Orlando, E., \& Strong, A. W. 2007, Ap\&SS, 309, 359

Pillitteri, I., Wolk, S. J., \& Megeath, S. T. 2016, ApJ, 820, L28

Prest, M., Barbiellini, G., Bordignon, G., et al. 2003, Nucl. Instrum. Methods Phys. Res. A, 501, 280

Rauch, B. F., Link, J. T., Lodders, K., et al. 2009, ApJ, 697, 2083

Sabatini, S., Donnarumma, I., Tavani, M., et al. 2015, ApJ, 809, 60

Schlafly, E. F., Green, G., Finkbeiner, D. P., et al. 2014, ApJ, 789, 15

Schlegel, D. J., Finkbeiner, D. P., \& Davis, M. 1998, ApJ, 500, 525

Tavani, M., Barbiellini, G., Argan, A., et al. 2009, A\&A, 502, 995

van Leeuwen, F. 2007, A\&A, 474, 653

Voelk, H. J., \& Forman, M. 1982, ApJ, 253, 188 